\title{
New Dimensions from Gauge-Higgs Unification
}

\author{
Yutaka Hosotani* \\ Department of Physics, Osaka University, Toyonaka, Osaka 560-0043, Japan \\ E-mail: hosotanidhet.phys.sci.osaka-u.ac.jp
}

The Higgs boson is unified with gauge fields in the gauge-Higgs unification. The $S O(5) \times U(1)$ gauge-Higgs electroweak unification in the Randall-Sundrum warped space yields almost the same phenomenology at low energies as the standard model, and gives many predictions for the Higgs couplings and new $W^{\prime}, Z^{\prime}$ bosons around $6 \sim 8 \mathrm{TeV}$, which can be tested at $14 \mathrm{TeV} \mathrm{LHC}$. The gauge-Higgs grand unification is achieved in $S O(11)$ gauge theory. It suggests the existence of the sixth dimension (GUT dimension) in addition to the fifth dimension (electroweak dimension). The proton decay is naturally suppressed in the gauge-Higgs grand unification.

Corfu Summer Institute 2016 "School and Workshops on Elementary Particle Physics and Gravity" 31 August - 23 September, 2016

Corfu, Greece

${ }^{*}$ Speaker. 


\section{Standard model}

The Standard Model (SM) is very successful at low energies. It is gauge theory of $S U(3)_{C} \times$ $S U(2)_{L} \times U(1)_{Y}$, whose Lagrangian density consists of four parts;

$$
\begin{aligned}
\mathscr{L} & =\mathscr{L}_{\text {gauge }}+\mathscr{L}_{\text {Higgs }}+\mathscr{L}_{\text {fermion }}+\mathscr{L}_{\text {Yukawa }}, \\
\mathscr{L}_{\text {gauge }} & =-\frac{1}{2} \operatorname{Tr} G_{\mu v} G^{\mu \nu}-\frac{1}{2} \operatorname{Tr} F_{\mu v} F^{\mu \nu}-\frac{1}{4} B_{\mu v} B^{\mu \nu}, \\
\mathscr{L}_{\text {Higgs }} & =\left|D_{\mu} \Phi\right|^{2}-V[\Phi], \\
\mathscr{L}_{\text {fermion }} & =\sum \bar{\psi}_{j} i \gamma^{\mu} D_{\mu} \psi_{j}, \\
\mathscr{L}_{\text {Yukawa }} & =\sum\left\{y_{j k}^{d} \bar{\psi}_{j} \Phi \psi_{k}+y_{j k}^{u} \bar{\psi}_{j} \tilde{\Phi} \psi_{k}\right\}+(\text { h.c. }) .
\end{aligned}
$$

The form of the parts $\mathscr{L}_{\text {gauge }}$ and $\mathscr{L}_{\text {fermion }}$ is determined by the gauge principle, and is beautiful. The form of the Higgs potential $V[\Phi]$ in $\mathscr{L}_{\text {Higgs }}$, however, is given in ad hoc manner. The Yukawa couplings $y_{j k}^{u, d}$ in $\mathscr{L}_{\text {Yukawa }}$ are arbitrary as well. The parts $\mathscr{L}_{\text {Higgs }}$ and $\mathscr{L}_{\text {Yukawa }}$ lack a principle.

The electroweak (EW) gauge symmetry breaking in the SM is brought about by an intentional choice of $V[\Phi]$ which is assumed to have a global minimum at $\langle\Phi\rangle \neq 0$. In other words, the EW gauge symmetry breaking is enforced by hand. The Higgs boson remains mysterious in the SM.

\section{Gauge-Higgs unification}

In the gauge-Higgs unification one starts with gauge theory in higher dimensions. [U, [1], []] The Higgs field becomes a part of the extra-dimensional component of gauge fields. Schematically

$$
\begin{gathered}
\mathscr{L}_{\text {gauge }}+\mathscr{L}_{\text {Higgs }} \Longrightarrow \mathscr{L}_{\text {gauge }}^{5 \mathrm{~d}}, \\
\mathscr{L}_{\text {fermion }}+\mathscr{L}_{\text {Yukawa }} \Longrightarrow \mathscr{L}_{\text {fermion }}^{5 \mathrm{~d}} .
\end{gathered}
$$

The effective Higgs potential is generated dynamically at the quantum level from $\mathscr{L}_{\text {gauge }}^{5 \mathrm{~d}}+\mathscr{L}_{\text {fermion }}^{5 \mathrm{~d}}$. In short, the theory is governed by the gauge principle, and becomes concise and beautiful. [⿴囗十, []]

In the gauge-Higgs unification in five dimensions $\left(x^{\mu}, y\right)$

$$
A_{M}=\left\{\begin{array}{l}
A_{\mu} \supset \gamma, W, Z \\
A_{y} \supset \text { Higgs boson } \sim \text { Aharonov-Bohm }(\mathrm{AB}) \text { phase } \theta_{H}
\end{array}\right.
$$

When the fifth dimension is not simply connected, the Higgs field appears as an Aharonov-Bohm phase $\theta_{H}$ in the fifth dimension. The effective potential $V_{\text {eff }}\left(\theta_{H}\right)$ becomes nontrivial at the one-loop level. When $V_{\text {eff }}\left(\theta_{H}\right)$ is minimized at $\theta_{H} \neq 0$, the EW symmetry is dynamically broken. Finite Higgs boson mass is generated. The gauge-hierarchy problem is solved.

\section{3. $S O(5) \times U(1)$ gauge-Higgs $\mathbf{E W}$ unification}

The Randall-Sundrum (RS) warped space is specified with the metric

$$
d s^{2}=e^{-2 \sigma(y)} d x^{\mu} d x_{\mu}+d y^{2},
$$




$$
\begin{aligned}
& \sigma(y)=\sigma(-y)=\sigma(y+2 L), \\
& \sigma(y)=k|y| \quad \text { for }|y| \leq L .
\end{aligned}
$$

The RS space has topology of $M^{4} \times\left(S^{1} / Z_{2}\right)$, in which $\left(x^{\mu}, y\right),\left(x^{\mu},-y\right)$ and $\left(x^{\mu}, y+2 L\right)$ are identified. Its fundamental region is $5 \mathrm{~d}$ AdS space sandwiched by UV and IR branes, at $y_{0}=0$ and $y_{1}=L$. The $5 \mathrm{~d}$ cosmological constant is given by $\Lambda=-6 k^{2}$. The $S O(5)$ and $U(1)_{X}$ gauge fields, $A_{M}$ and $B_{M}$, satisfy

$$
\begin{aligned}
& \left(\begin{array}{c}
A_{\mu} \\
A_{y}
\end{array}\right)\left(x, y_{j}-y\right)=P_{j}\left(\begin{array}{c}
A_{\mu} \\
-A_{y}
\end{array}\right)\left(x, y_{j}+y\right) P_{j}^{-1}, P_{j} \in S O(5), P_{j}^{2}=1, \\
& \left(\begin{array}{c}
B_{\mu} \\
B_{y}
\end{array}\right)\left(x, y_{j}-y\right)=\left(\begin{array}{c}
B_{\mu} \\
-B_{y}
\end{array}\right)\left(x, y_{j}+y\right),
\end{aligned}
$$

Although gauge potentials themselves are not single-valued, physical gauge-invariant quantities

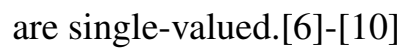

The set of the matrices $P_{0}, P_{1}$ is called the orbifold boundary condition. We take

$$
P_{0}=P_{1}=\left(\begin{array}{ccccc}
-1 & & & & \\
& -1 & & & \\
& & -1 & & \\
& & & -1 & \\
& & & & +1
\end{array}\right),
$$

by which gauge symmetry $\mathscr{G}=S O(5) \times U(1)_{X}$ is reduced to $\mathscr{H}=S O(4) \times U(1)_{X}$. Zero modes (parity even-even modes) appear in the $\mathscr{H}$ part of $A_{\mu}, B_{\mu}$, and in the $\mathscr{G} / \mathscr{H}$ part of $A_{y}$. The latter is an $S O(4) \simeq S U(2)_{L} \times S U(2)_{R}$ vector, or an $S U(2)_{L}$ doublet, corresponding to the $4 \mathrm{~d}$ Higgs field in the SM.

Quark-lepton multiplets are introduced in the vector representation of $S O(5)$ in the bulk. In addition, one introduces dark fermions in the spinor representation in the bulk. On the UV brane at $y=0$ brane fermions in $S U(2)_{L}$ doublet and a brane scalar $\Phi$ in $S U(2)_{R}$ doublet are introduced. The brane scalar $\Phi$ spontaneously breaks $S U(2)_{R} \times U(1)_{X}$ to $U(1)_{Y}$, and at the same time gives rise to additional mass terms for fermions. The resultant spectrum at low energies $(<1 \mathrm{TeV})$ is that of the SM. The effective potential $V_{\text {eff }}\left(\theta_{H}\right)$ is evaluated at the one loop. Contributions from the top quark multiplet and dark fermions triggers the EW gauge symmetry breaking with a Higgs boson mass $m_{H}=125 \mathrm{GeV}$.

\section{Success}

The $S O(5) \times U(1)_{X}$ gauge-Higgs unification is successful. The gauge principle governs the theory, including dynamics of the 4d Higgs boson.[10]-[24]

(1) The $4 \mathrm{~d}$ Higgs boson, the four-dimensional fluctuation mode of the $\mathrm{AB}$ phase $\theta_{H}$ in the fifth dimension, is massless at the tree level but acquires a nonvanishing mass at the one loop level 
which is free from divergence and independent of regularization methods employed. The gauge hierarchy problem, a cumbersome problem in many theories, is naturally solved.

(2) The phenomenology at low energies $(\leq 1 \mathrm{TeV})$ for $\theta_{H}<0.1$ is almost the same as in the SM.

(3) There is no vacuum instability problem associated with the $4 \mathrm{~d}$ Higgs scalar field.[25] The effective potential for the $4 \mathrm{~d}$ Higgs field $H(x)$ is given by $V_{\text {eff }}\left(\theta_{H}+H / f_{H}\right)$. The large gauge invariance guarantees the periodicity $V_{\text {eff }}\left(\theta_{H}+2 \pi\right)=V_{\text {eff }}\left(\theta_{H}\right)$, which in turn implies that there never occurs the instability. It has been explicitly shown that $V_{\text {eff }}\left(\theta_{H}\right)$ is finite at the one loop level.

(4) Dynamical EW symmetry breaking takes place in the RS space. The existence of a heavy quark, the top quark $m_{t}>m_{W}$, is crucial . $V_{\text {eff }}\left(\theta_{H}\right)$ is controlled by the $W$ and $Z$ bosons, the top quark multiplet, and the dark fermions. Light quarks and leptons multiplets are irrelevant for the EW symmetry breaking in the RS space.

\section{Predictions}

The gauge-Higgs unification gives many predictions to be confirmed by the forthcoming and future experiments. Although the model contains several parameters, most of physical quantities are determined by the $\mathrm{AB}$ phase $\theta_{H}$.

(a) The Yukawa couplings of quarks and leptons, $Y_{\alpha}$, the three-point couplings of the Higgs boson

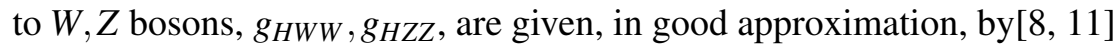

$$
Y_{\alpha}, g_{H W W}, g_{H Z Z} \simeq(\mathrm{SM} \text { values }) \times \cos \theta_{H} .
$$

The deviation from the SM is less than $1 \%$ for $\theta_{H}<0.1$.

(b) Decay of the Higgs boson to $\gamma \gamma, Z \gamma$, and two gluons take place through one-loop diagrams. In the gauge-Higgs unification an infinite number of various Kaluza-Klein (KK) modes run inside the loop. (Fig. W) Each of their contributions gives $O(1 / n)$ correction to the decay width where $n$ is the KK number. There appears miraculous cancellation among them so that the sum of all contributions turns out finite and small. It gives less than $1 \%$ correction to those in which SM particles run inside the loop for $\theta_{H}<0.1$. The cancellation in the process $H \rightarrow Z \gamma$ is highly nontrivial, as the KK number can change inside the loop.[ए], [1]]
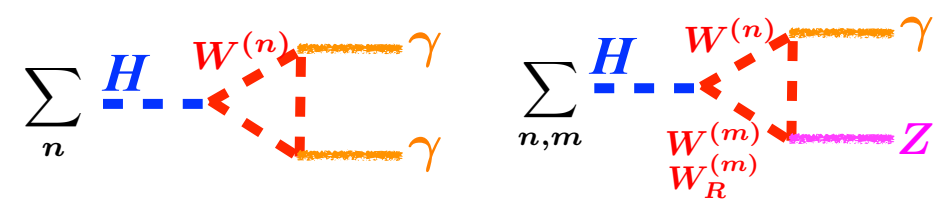

Figure 1: Diagrams contributing to $H \rightarrow \gamma \gamma, Z \gamma$. The infinite sums converge and give small corrections to the SM. There are diagrams in which the top quark and its KK tower run inside the loops.

(c) An immediate consequence of (a) and (b) is that both the production rate of the Higgs boson at LHC and decay widths of the Higgs boson to various modes are all suppressed in good approximation by a factor $\cos ^{2} \theta_{H}$ compared to those in the SM. Branching fractions of various decay modes 
are nearly the same as in the SM. The signal strengths of the various decay modes are suppressed by a factor $\cos ^{2} \theta_{H}$. For $\theta_{H}<0.1$ the deviation from the SM is less than $1 \%$.

(d) The Higgs cubic and quartic self-couplings, $\lambda_{3}^{H}, \lambda_{4}^{H}$, deviate from those in the SM, which can be tested in future. Although the model has several parameters to be fixed, many of physical quantities such as $\lambda_{3}^{H}, \lambda_{4}^{H}$, the KK mass scale $m_{\mathrm{KK}}$, and the masses of the first KK modes $\gamma^{(1)}, Z^{(1)}, W^{(1)}$ depend only on $\theta_{H}$ in very good approximation. This property is called the universality. (Fig. [])

The universality leads to strong prediction power in the gauge-Higgs unification. Suppose that the first KK mode $Z^{(1)}$ is found at $m_{0}$. From the relation $m_{Z^{(1)}}\left(\theta_{H}\right)=m_{0}$, the value $\theta_{H}$ is determined. Then other quantities $\lambda_{3}^{H}\left(\theta_{H}\right), \lambda_{4}^{H}\left(\theta_{H}\right), m_{W^{(1)}}\left(\theta_{H}\right)$ etc. are determined, and can be checked experimentally.
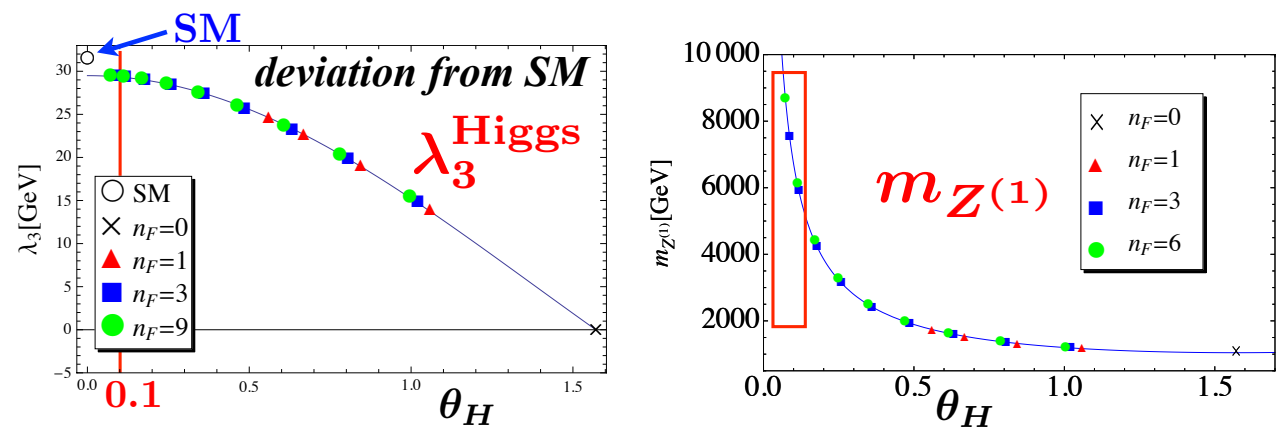

Figure 2: Universality. $\lambda_{3}^{H}, \lambda_{4}^{H}, m_{\mathrm{KK}}, m_{Z^{(1)}}, m_{\gamma^{(1)}}, m_{W^{(1)}}$ etc. are determined by $\theta_{H}$, almost independent of the details of the model. In particular, they do not depend on the number $n_{F}$ of dark fermions.

(e) The prediction of $Z^{\prime}$ events gives the cleanest test of the model. (Fig. (3) The first KK modes of the photon, $Z$ boson, and $Z_{R}$ boson appear as $Z^{\prime}$ events. $\left(Z_{R}\right.$ is associated with $S U(2)_{R}$, and has no zero mode.) For $\theta_{H}=0.114$, their masses are $\left(m_{Z_{R}^{(1)}}, m_{Z^{(1)}}, m_{\gamma^{(1)}}\right)=(5.73,6.07,6.08) \mathrm{TeV}$ and the widths are $\left(\Gamma_{Z_{R}^{(1)}}, \Gamma_{Z^{(1)}}, \Gamma_{\gamma^{(1)}}\right)=(482,342,886) \mathrm{GeV}$. For $\theta_{H}=0.073$, their masses are $\left(m_{Z_{R}^{(1)}}, m_{Z^{(1)}}, m_{\gamma^{(1)}}\right)=(8.00,8.61,8.61) \mathrm{TeV}$ and the widths are $\left(\Gamma_{Z_{R}^{(1)}}, \Gamma_{Z^{(1)}}, \Gamma_{\gamma^{(1)}}\right)=(553,494,1040)$ $\mathrm{GeV}$ [[24]

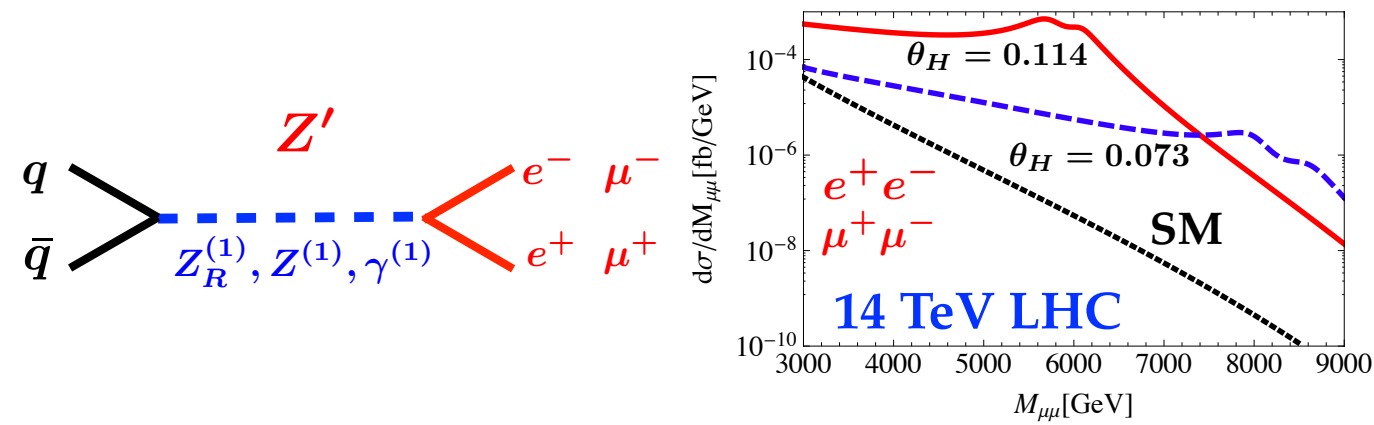

Figure 3: $Z^{\prime}$ production at LHC. 


\section{6. $S O(11)$ gauge-Higgs grand unification}

It is necessary to incorporate strong interactions in the framework of gauge-Higgs unification. This leads to gauge-Higgs grand unification.[[26]-[37] We look for a scenario in which the EW Higgs boson appears as the extra-dimensional component of gauge potentials, and electromagnetic, weak, and strong interactions are unified in a single group, and no exotic particles appears at low energies.

One might think that the gauge group should contain $S O(5) \times U(1)_{X} \times S U(3)_{C}$ as a subgroup. This turns out not to be the case. It is seen that $S O(11)$ gauge theory does a job, keeping good features of the $S O(5) \times U(1)_{X}$ gauge-Higgs EW unification. [34, [36]

One starts with $\mathscr{G}=S O(11)$ gauge theory in the Randall-Sundrum warped space (B.D). The orbifold boundary condition is given by

$$
\begin{array}{ll}
P_{0}^{\mathrm{vec}}=\operatorname{diag}\left(I_{10},-I_{1}\right), & P_{1}^{\mathrm{vec}}=\operatorname{diag}\left(I_{4},-I_{7}\right), \\
P_{0}^{\mathrm{sp}}=I_{16} \otimes \sigma^{3}, & P_{1}^{\mathrm{sp}}=I_{2} \otimes \sigma^{3} \otimes I_{8}
\end{array}
$$

in vectorial and spinorial representations. At the UV brane $S O(11)$ is broken to $S O(10)$ by $P_{0}$, whereas at the IR brane it is broken to $S O(4) \times S O(7)$. As a whole $\mathscr{G}=S O(11)$ is broken to $\mathscr{H}=S O(4) \times S O(6)$. Note that $S O(4) \simeq S U(2)_{L} \times S U(2)_{R}$, and $S O(6) \simeq S U(4)$. At this stage $A_{\mu}$ has zero modes in the block $\mathscr{H}$. On the other hand $A_{y}$ has zero modes in the block $[\mathscr{G} / S O(10)] \cap$ $[\mathscr{G} / S O(4) \times S O(7)]$. In the vectorial representation $A_{y}$ has zero modes in the components $A_{y}^{a 11}$ $(a=1 \sim 4$ ), which correspond to the 4d Higgs field in the SM. (Fig. 目)
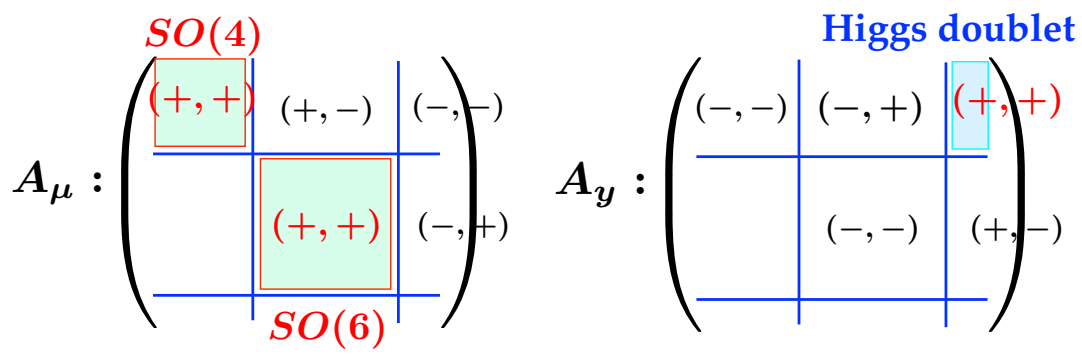

Figure 4: $S O(11)$ gauge-Higgs grand unification. Parity $\left(P_{0}, P_{1}\right)=(+,+)$ modes appear in the $S O(4) \times$ $S O(6)$ block of $A_{\mu}$ and in the $[S O(11) / S O(10)] \cap[S O(11) / S O(4) \times S O(7)]$ block of $A_{y}, A_{y}^{a 11}(a=1 \sim 4)$.

On the UV brane a brane scalar $\Phi_{16}$ is introduced. $\Phi_{16}$ spontaneously breaks $S O(10)$ to $S U(5)$. As a result $\mathscr{G}=S O(11)$ is reduced to $\mathscr{G}_{\mathrm{SM}}=S U(2)_{L} \times U(1)_{Y} \times S U(3)_{C}$. Note that $S U(3)_{C} \subset S O(6)$, and that $U(1)_{Y}$ is a combination of $S U(2)_{R}$ and $S O(6)$. $\mathscr{G}_{S M}$ is dynamically broken to $U(1)_{\mathrm{EM}} \times S U(3)_{C}$ through the Hosotani mechanism. The Weinberg angle at the GUT scale becomes $\sin ^{2} \theta_{W}=\frac{3}{8}$, the same value as in the $S U(5)$ or $S O(10)$ GUT in four dimensions. See the comparison of gauge-Higgs EW and grand unification in Fig. [1].

Fermions are introduced in the spinor $\left(\Psi_{\mathbf{3 2}}\right)$ and vector $\left(\Psi_{11}\right)$ representations of $S O(11) . \Psi_{\mathbf{3 2}}$, 


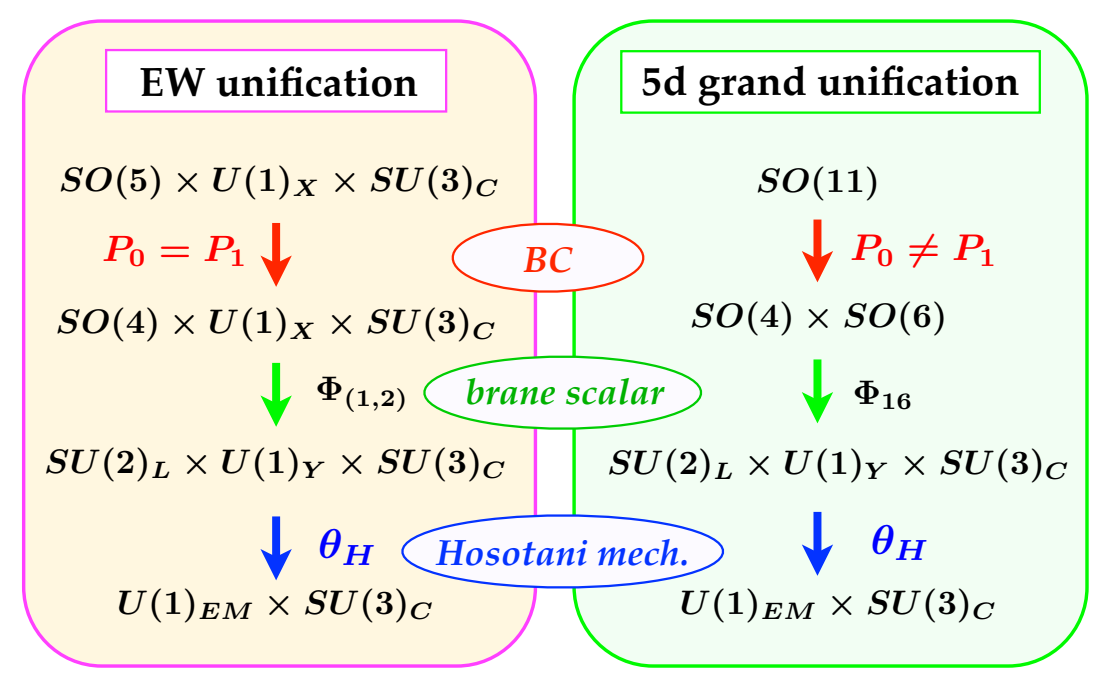

Figure 5: Comparison of gauge-Higgs EW and grand unification

for instance, satisfies $\Psi_{\mathbf{3 2}}\left(x, y_{j}-y\right)=-\gamma^{5} P_{j}^{\mathrm{sp}} \Psi_{\mathbf{3 2}}\left(x, y_{j}+y\right)$. The content of $\Psi_{\mathbf{3 2}}$ is given by

$$
\begin{gathered}
\Psi_{\mathbf{3 2}}=\left(\begin{array}{c}
\Psi_{\mathbf{1 6}} \\
\Psi_{\overline{\mathbf{1 6}}}
\end{array}\right), \Psi_{\mathbf{1 6}}=\left(\begin{array}{c}
v \\
e \\
\hat{e} \\
\hat{v} \\
u_{k} \\
d_{k} \\
\hat{d}_{k} \\
\hat{u}_{k}
\end{array}\right), \Psi_{\overline{\mathbf{1 6}}}=\left(\begin{array}{c}
v^{\prime} \\
e^{\prime} \\
\hat{e}^{\prime} \\
\hat{v}^{\prime} \\
u_{k}^{\prime} \\
d_{k}^{\prime} \\
\hat{d}_{k}^{\prime} \\
\hat{u}_{k}^{\prime}
\end{array}\right),(k=1 \sim 3), \\
\text { zero modes : } \quad\left(\begin{array}{c}
v_{L} \\
e_{L}
\end{array}\right),\left(\begin{array}{c}
u_{k L} \\
d_{k L}
\end{array}\right),\left(\begin{array}{c}
v_{R}^{\prime} \\
e_{R}^{\prime}
\end{array}\right),\left(\begin{array}{c}
u_{k R}^{\prime} \\
d_{k R}^{\prime}
\end{array}\right) .
\end{gathered}
$$

$\hat{e}, \hat{u}$, and $\hat{d}$ fields have charges $+1,-\frac{2}{3}$, and $+\frac{1}{3}$, respectively. Zero modes appear only for the components of quarks and leptons. Vector multiplets $\Psi_{\mathbf{1 1}}$ are introduced to reproduce the mass spectrum of down-type quarks and leptons.

One interesting feature is that all quarks and leptons appear in $\Psi_{32}$ as particles with the $\Psi$ fermion number $N_{\Psi}=+1 . N_{\Psi}$ is conserved even in the presence of $\Psi_{\mathbf{1 1}}$. A proton has $N_{\Psi}=3$, whereas $\pi^{0} e^{+}$has $N_{\Psi}=-1$. Thus the proton decay $p \rightarrow \pi^{0} e^{+}$is forbidden. This should be contrasted to the situation in the 4d GUT. In $S O(10)$ GUT in four dimensions a fermion multiplet is introduced in the spinor representation $\Psi_{\mathbf{1 6}}$ for left-handed fields. In the notation in (6.2), $\left(u_{k}, d_{k}\right) \rightarrow\left(u_{k L}, d_{k L}\right)$ and $\left(\hat{u}_{k}, \hat{d}_{k}\right) \rightarrow\left(u_{k L}^{c}, d^{c}{ }_{k L}\right)$. As $u^{c}{ }_{k L} \sim u_{k R}^{\dagger}$, gauge and Higgs interactions convert a particle to an anti-particle, which induces proton decay. In the gauge-Higgs grand unification such process is absent and the proton decay is naturally suppressed.

However, there is a problem. Careful examination reveals that in the first and second generations $\hat{u}, \hat{d}, \hat{e}$ have light masses, which contradicts the observation. The source of this difficulty lies in the fact that the parity at $y_{0}=0$ and $y_{1}=L$ is (even, odd) or (odd, even) for $\hat{u}, \hat{d}, \hat{e}$. In the RS 
warped space it leads to light masses. In other words, $P_{0} \neq P_{1}$ in the RS warped space gives rise to a trouble.

\section{Gauge-Higgs grand unification in six dimensions}

The difficulty is solved in gauge-Higgs unification in six-dimensional hybrid-warped space.[38] Consider the six-dimensional space with a metric

$$
\begin{aligned}
& d s^{2}=e^{-2 \sigma(y)}\left(d x^{\mu} d x_{\mu}+d v^{2}\right)+d y^{2}, \\
& \sigma(y)=\sigma(-y)=\sigma\left(y+2 L_{5}\right), \\
& \sigma(y)=k|y| \text { for }|y| \leq L_{5} .
\end{aligned}
$$

We identify points

$$
\begin{aligned}
\left(x^{\mu}, y, v\right) & \sim\left(x^{\mu}, y+2 L_{5}, v\right) \sim\left(x^{\mu}, y, v+2 \pi R_{6}\right) \\
& \sim\left(x^{\mu},-y,-v\right) .
\end{aligned}
$$

The spacetime has topology of $M^{4} \times\left(T^{2} / Z_{2}\right)$. The fundamental region can be taken as $\{0 \leq y \leq$ $\left.L_{5}, 0 \leq v<2 \pi R_{6}\right\}$ The metric $(\mathbb{C})$ solves the Einstein equation with five-dimensional branes at $y=0$ and $y=L_{5}$. Six-dimensional spacetime is an AdS space with $\Lambda=-10 k^{2}$. The sixth dimension is curled up in a circle with a very small radius $R_{6}$. We suppose that $z_{L}=e^{k L_{5}} \gg 1$ and

$$
m_{\mathrm{KK}_{5}}=\frac{\pi k}{e^{k L_{5}}-1} \sim \pi k e^{-k L_{5}} \ll m_{\mathrm{KK}_{6}}=\frac{1}{R_{6}} .
$$

Under $Z_{2}$ parity $(y, v) \rightarrow(-y,-v)$, there appear four fixed points. (See Fig. 6 .)

$$
\left(y_{0}, v_{0}\right)=(0,0),\left(y_{1}, v_{1}\right)=\left(L_{5}, 0\right),\left(y_{2}, v_{2}\right)=\left(0, \pi R_{6}\right),\left(y_{3}, v_{3}\right)=\left(L_{5}, \pi R_{6}\right) .
$$

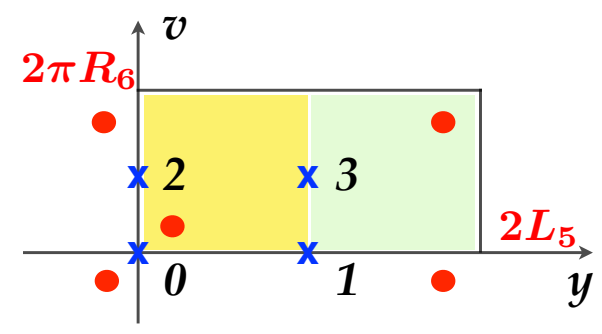

Figure 6: Four fixed points (in blue) in the 6 dimensional gauge-Higgs grand unification.The fundamental region is given by $0 \leq y \leq L_{5}, 0 \leq v<2 \pi R_{6}$. Red circle points represent a single spacetime point. Around each fixed point, parity is defined.

We consider $S O(11)$ gauge theory in the 6-dimensional hybrid-warped space (ㅁ. $)$. Gauge potentials $A_{M}$ satisfy

$$
\left(\begin{array}{c}
A_{\mu} \\
A_{y} \\
A_{v}
\end{array}\right)\left(x, y_{j}-y, v_{j}-v\right)=P_{j}\left(\begin{array}{c}
A_{\mu} \\
-A_{y} \\
-A_{v}
\end{array}\right)\left(x, y_{j}+y, v_{j}+v\right) P_{j}^{-1}
$$




$$
P_{j} \text { or }-P_{j} \in S O(11), P_{j}^{2}=1, P_{3}=P_{1} P_{0} P_{2}=P_{2} P_{0} P_{1} .
$$

Note that only three of the four $P_{j}$ 's are independent, and the condition $P_{1} P_{0} P_{2}=P_{2} P_{0} P_{1}$ must be satisfied for the consistency. We take, in place of (6.d),

$$
\begin{aligned}
& P_{0}^{\mathrm{vec}}=P_{1}^{\mathrm{vec}}=\operatorname{diag}\left(I_{4},-I_{7}\right), \\
& P_{2}^{\mathrm{vec}}=P_{3}^{\mathrm{vec}}=\operatorname{diag}\left(I_{10},-I_{1}\right) .
\end{aligned}
$$

Fermion multiplets $\Psi_{\mathbf{3 2}}$ and $\Psi_{\mathbf{1 1}}$ are introduced in the bulk. $\Psi_{\mathbf{3 2}}$ is a $6 \mathrm{~d}$ Weyl fermion, and satisfies $\Psi_{\mathbf{3 2}}\left(x, y_{j}-y, v_{j}-v\right)=P_{j}^{\mathrm{sp}} \bar{\gamma} \Psi_{\mathbf{3 2}}\left(x, y_{j}+y, v_{j}+v\right)$ where $\bar{\gamma}=-i \Gamma^{5} \Gamma^{6}$. With this boundary condition zero modes appear chiral, with the quark-lepton content given in (6.2). Furthermore, the lightest modes of hat fields $\hat{e}, \hat{d}, \hat{u}$ etc. have large masses of $O\left(R_{6}^{-1}\right)$.

The symmetry breaking pattern is similar to the five-dimensional case. The orbifold boundary condition in the sixth dimension reduces $S O(11)$ to $S O(10)$, and the condition in the fifth dimension reduces $S O(11)$ to $S O(4) \times S O(7)$. A brane scalar $\Phi_{\mathbf{3 2}}(x, v)$ is introduced on the five-dimensional UV brane at $y=0$. It spontaneously breaks $S O(11)$ to $S U(5)$. As a result the SM symmetry $\mathscr{G}_{S M}=S U(2)_{L} \times U(1)_{Y} \times S U(3)_{C}$ survives. By the Hosotani mechanism the symmetry is further broken to $U(1)_{\mathrm{EM}} \times S U(3)_{C}$. Zero modes of $A_{y}$ correspond to the $4 \mathrm{~d}$ Higgs doublet. There appear zero modes of $A_{v}$ in the same $S O(11)$ components as $A_{y}$, which acquire masses of order $g R_{6}^{-1}$ by the Hosotani mechanism.

\section{Summary}

The gauge-Higgs unification is promising. The $S O(5) \times U(1)$ gauge-Higgs EW unification gives definitive predictions to be tested in the forthcoming LHC experiments. The incorporation of strong interactions leads to the $S O(11)$ gauge-Higgs grand unification. It seems necessary to introduce the sixth dimension to have a spectrum consistent at low energies. The fifth dimension serves as an EW dimension, whereas the sixth dimension as a GUT dimension. We are entering into an era of "New Dimensions".

\section{Acknowledgement}

This work was supported in part by the Japan Society for the Promotion of Science, Grantsin-Aid for Scientific Research No 15K05052.

\section{References}

[1] Y. Hosotani, Dynamical Mass Generation by Compact Extra Dimensions, Phys. Lett. B126, 309 (1983); Dynamics of Nonintegrable Phases and Gauge Symmetry Breaking, Ann. Phys. (N.Y.) 190, 233 (1989).

[2] A. T. Davies and A. McLachlan, Gauge group breaking by Wilson loops, Phys. Lett. B200, 305 (1988); Congruency class effects in the Hosotani model, Nucl. Phys. B317, 237 (1989).

[3] H. Hatanaka, T. Inami and C.S. Lim, The gauge hierarchy problem and higher dimensional gauge theories, Mod. Phys. Lett. A13, 2601 (1998). 
[4] Y. Hosotani and N. Yamatsu, Gauge-Higgs Grand Unification, PoS PLANCK2015, 058 (2015). arXiv:1511.01674 [hep-ph].

[5] Y. Hosotani, Gauge-Higgs EW and Grand Unification, Int. J. Mod. Phys. A31, 1630031 (2016). arXiv:1606.08108 [hep-ph].

[6] K. Agashe, R. Contino and A. Pomarol, The Minimal Composite Higgs Model, Nucl. Phys. B719, 165 (2005).

[7] A. D. Medina, N. R. Shah, and C. E. Wagner, Gauge-Higgs Unification and Radiative Electroweak Symmetry Breaking in Warped Extra Dimensions, Phys. Rev. D76, 095010 (2007).

[8] Y. Hosotani and Y. Sakamura, Anomalous Higgs couplings in the $S O(5) \times U(1)_{B-L}$ gauge-Higgs unification in warped spacetime, Prog. Theoret. Phys. 118, 935 (2007).

[9] Y. Hosotani, K. Oda, T. Ohnuma and Y. Sakamura, Dynamical Electroweak Symmetry Breaking in $S O(5) \times U(1)$ Gauge-Higgs Unification with Top and Bottom Quarks, Phys. Rev. D78, 096002 (2008); Erratum-ibid. D79, 079902 (2009).

[10] S. Funatsu, H. Hatanaka, Y. Hosotani, Y. Orikasa, and T. Shimotani, Novel universality and Higgs decay $H \rightarrow \gamma \gamma, g g$ in the $S O(5) \times U(1)$ gauge-Higgs unification, Phys. Lett. B722, 94 (2013).

[11] Y. Hosotani and Y. Kobayashi, Yukawa couplings and effective interactions in gauge-Higgs unification, Phys. Lett. B674, 192 (2009).

[12] N. Haba, Y. Sakamura and T. Yamashita, Tree-level unitarity in Gauge-Higgs Unification, JHEP 1003, 069 (2010).

[13] Y. Hosotani, S. Noda and N. Uekusa, The Electroweak gauge couplings in $S O(5) \times U(1)$ gauge-Higgs unification, Prog. Theoret. Phys. 123, 757 (2010).

[14] Y. Hosotani, M. Tanaka, and N. Uekusa, Collider signatures of the $S O(5) \times U(1)$ gauge-Higgs unification, Phys. Rev. D84, 075014 (2011).

[15] M. Kakizaki, S. Kanemura, H. Taniguchi and T. Yamashita Higgs sector as a probe of supersymmetric grand unification with the Hosotani mechanism, Phys. Rev. D89, 075013 (2014).

[16] N. Kurahashi, C.S. Lim, K. Tanabe, Anomalous Higgs interactions in dimensional deconstruction, Prog. Theoret. Exp. Phys. 2014, 123B04 (2014).

[17] S. Funatsu, H. Hatanaka, Y. Hosotani, Y. Orikasa and T. Shimotani, LHC signals of the $S O(5) \times U(1)$ gauge-Higgs unification, Phys. Rev. D89, 095019 (2014).

[18] S. Funatsu, H. Hatanaka, Y. Hosotani, Y. Orikasa and T. Shimotani, Dark matter in the $S O(5) \times U(1)$ gauge-Higgs unification, Prog. Theoret. Exp. Phys. 2014, 113B01 (2014).

[19] S. Funatsu, H. Hatanaka and Y. Hosotani, $H \rightarrow Z \gamma$ in the gauge-Higgs unification, Phys. Rev. D92, 115003 (2015).

[20] N. Kitazawa and Y. Sakai, Constraints on gauge-Higgs unification models at the LHC, Mod. Phys. Lett. A31, 1650041 (2016).

[21] Y. Matsumoto and Y. Sakamura, Yukawa couplings in $6 D$ gauge-Higgs unification on $T^{2} / Z_{N}$ with magnetic fluxes, Prog. Theoret. Exp. Phys. 2016, $053 \mathrm{~B} 06$ (2016).

[22] Y. Adachi and N. Maru, Trilinear gauge boson couplings in the gauge-Higgs unification, Prog. Theoret. Exp. Phys. 2016, $073 \mathrm{~B} 06$ (2016).

[23] K. Hasegawa and C.S. Lim, A few comments on the Higgs boson decays in gauge-Higgs unification, Phys. Rev. D94, 055021 (2016).

[24] S. Funatsu, H. Hatanaka, Y. Hosotani and Y. Orikasa, Collider signals of $W^{\prime}$ and $Z^{\prime}$ bosons in the gauge-Higgs unification, Phys. Rev. D95, 035031 (2017). 
[25] G. Degrassi, S. Di Vita, J. Elias-Miro, J.R. Espinosa, G.F. Giudice, G. Isidori, A. Strumia, Higgs mass and vacuum stability in the Standard Model at NNLO, JHEP 08, 098 (2012).

[26] G. Burdman and Y. Nomura, Unification of Higgs and gauge fields in five dimensions, Nucl. Phys. B656, 3 (2003).

[27] N. Haba, Masatomi Harada, Y. Hosotani and Y. Kawamura, Dynamical rearrangement of gauge symmetry on the orbifold $S^{1} / Z_{2}$, Nucl. Phys. B657, 169 (2003); Erratum-ibid. B669, 381 (2003).

[28] N. Haba, Y. Hosotani, Y. Kawamura and T. Yamashita, Dynamical symmetry breaking in gauge Higgs unification on orbifold, Phys. Rev. D70, 015010 (2004).

[29] C.S. Lim and N. Maru, Towards a realistic grand gauge-Higgs unification, Phys. Lett. B653, 320 (2007).

[30] K. Kojima, K. Takenaga and T. Yamashita, Grand gauge-Higgs unification, Phys. Rev. D84, 051701(R) (2011).

[31] M. Frigerio, J. Serra and A. Varagnolo, Composite GUTs: models and expectations at the LHC, JHEP 1106, 029 (2011).

[32] K. Yamamoto, The formulation of gauge-Higgs unification with dynamical boundary conditions, Nucl. Phys. B883, 45 (2014).

[33] F. J. de Anda, Left-right model from gauge-Higgs unification with dark matter, Mod. Phys. Lett. A30, 1550063 (2015).

[34] Y. Hosotani and N. Yamatsu, Gauge-Higgs grand unification, Prog. Theoret. Exp. Phys. 2015, 111B01 (2015).

[35] N. Yamatsu, Gauge coupling unification in gauge-Higgs grand unification, Prog. Theoret. Exp. Phys. 2016, 043B02 (2016).

[36] A. Furui, Y. Hosotani and N. Yamatsu, Toward realistic gauge-Higgs grand unification, Prog. Theoret. Exp. Phys. 2016, $093 \mathrm{~B} 01$ (2016).

[37] K. Kojima, K. Takenaga and T. Yamashita, Gauge symmetry breaking patterns in an SU(5) grand gauge-Higgs unification, Phys. Rev. D95, 015021 (2017).

[38] Y. Hosotani and N. Yamatsu, in preparation. 